\title{
Residence time distribution for electrokinetic flow through a microchannel comprising a bundle of cylinders
}

\author{
Jyh-Ping Hsu ${ }^{\mathrm{a}, *}$, Chung-Chieh Ting ${ }^{\mathrm{a}}$, Duu-Jong Lee ${ }^{\mathrm{a}}$, Shiojenn Tseng ${ }^{\mathrm{b}}$, Chur-Jen Chen ${ }^{\mathrm{c}}, \mathrm{Ay} \mathrm{Su}^{\mathrm{d}}$ \\ a Department of Chemical Engineering, National Taiwan University, Taipei 10617, Taiwan \\ ${ }^{\mathrm{b}}$ Department of Mathematics, Tamkang University, Tamsui, Taipei 25137, Taiwan \\ ${ }^{\mathrm{c}}$ Department of Mathematics, Tunghai University, Taichung 403, Taiwan \\ d Department of Mechanical Engineering \& Fuel Cells Research Center, Yuan Ze University, 135 Yuan-Tung Road, Chung Li, Tao Yuan 320, Taiwan
}

Received 10 October 2006; accepted 16 November 2006

Available online 21 December 2006

\begin{abstract}
The electrokinetic flow of an electrolyte solution through a microchannel that comprises a bundle of cylinders is investigated for the case of constant surface potential. The system under consideration is simulated by a unit cell model, and analytical expressions for the flow field and the corresponding residence time distribution under various conditions are derived. These results are readily applicable to the assessment of the performance of a microreactor such as that which comprises a bundle of optical fibers. Numerical simulations are conducted to investigate the influences of the key parameters, including the thickness of the double layer, the strength of the applied electric field, the magnitude of the applied pressure gradient, and the characteristic sizes of a microchannel, on the residence time distribution. We show that the following could result in a shorter residence time: thin double layer, strong applied electric field, large applied pressure gradient, and small number of cylinders. Based on the thickness of the double layer, criteria are proposed for whether the flow field can be treated as a laminar flow or as a plug flow, two basic limiting cases in reactor design.
\end{abstract}

(C) 2006 Elsevier Inc. All rights reserved.

Keywords: Residence time distribution; Microchannel; Bundle of cylinders; Electrokinetic flow; Cell model

\section{Introduction}

An exact assessment of the performance of a reactor rests on knowledge of the precise velocity and local rate of mixing of every element of fluid inside. Unfortunately, this is nontrivial, if not impossible, even for vessels of simple geometry under drastic conditions. In fact, measuring the velocity and concentration at each point of a vessel is difficult and essentially infeasible, not to mention predicting them theoretically. In practice, this difficulty is partially circumvented by measuring or predicting the residence time distribution of the fluid elements as they flow through a vessel. Coupled with an appropriate local mixing model, the residence time distribution can then be used to predict the conversion of reactants as they pass through a reactor. For macroscaled reactors, residence time distributions can

\footnotetext{
* Corresponding author. Fax: +886223623040.

E-mail address: jphsu@ntu.edu.tw (J.-P. Hsu).
}

be derived analytically only for a very limited number of idealized vessels [1-3].

Modern chemical engineering technology encourages using processes that have low risks, consume low energy, and produce small amounts of wastes. One of the possible ways to achieve this is to reduce the size of reactors and produce products of high additive value $[4,5]$. Reactors the linear size of which is on the order of micrometers have been used successfully in the chemical and biomedical industries.

For the flow of fluid through a macrochannel, the pressure gradient is usually the main driving force. This might not be the case for a microchannel, where both a pressure gradient and an applied electric field can play a role. In the latter the direction of fluid flow can be adjusted by varying the charged conditions on the surface of a microchannel. Although the flow of fluid driven by an applied electric field in a microchannel has drawn the attention of many theoreticians and experimentalists in the last decade [6-12], available results for the corresponding resi- 
dence time distribution are still very limited. For the case where a microchannel plays the role of a reactor, knowledge of this distribution is essential to its design and optimization [1-3].

In a recent study we derived the residence time distribution for the electrokinetic flow in a long cylindrical microchannel [13]. In this study, the electrokinetic flow of an electrolyte solution through a microchannel that comprises a bundle of cylinders is analyzed under the condition of constant surface potential. It simulates, for example, a bundle of optical fiber reactors where liquid reactants flow through the space between optical fibers, the surface of which is coated with a photonactivated catalyst. A unit cell model where a microchannel is simulated by a representative cylinder surrounded by a concentric liquid shell, which is widely adopted in the description of concentrated dispersions of colloidal entities, is used. The residence time distributions under various conditions are derived, and the influences of the key parameters of the system under consideration on that distribution are discussed.

\section{Theory}

Let us consider the steady flow of an electrolyte solution through a microchannel that comprises a bundle of cylinders, illustrated in Fig. 1a. Here, a unit cell model is adopted, and as shown in Fig. 1b, the microchannel is simulated by a unit cell, which comprises a representative cylinder of length $L$ and diameter $2 a$ and a concentric liquid shell of diameter $2 \alpha a$, where

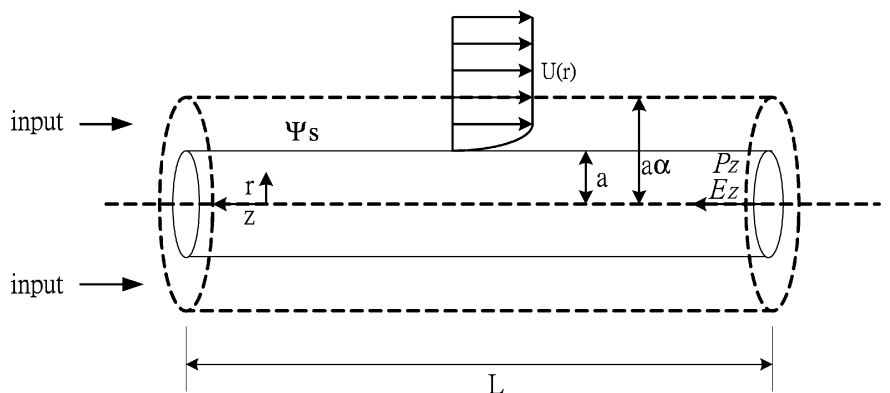

(a)

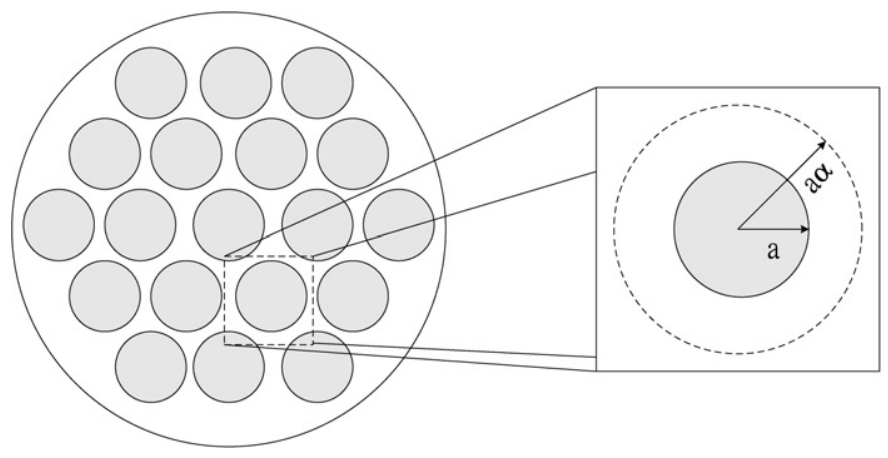

(b)

Fig. 1. The problem considered where an electrolyte solution flows through a microchannel comprising a bundle of cylinders with length $L$ driven by a pressure gradient $P_{z}$ and an electric field $E_{z}$ in the $z$-direction. The system is simulated by a representative cell comprising a cylinder of radius $a$ and a concentric liquid shell of radius $\alpha a$. $\psi$ is the surface potential, $(r, \theta, z)$ are the cylindrical coordinates with their origin located at the axis of the representative cylinder, and $U(r)$ is the radial velocity distribution. $\left[\left(\alpha^{2}-1\right) / \alpha^{2}\right]$ is a measure of the volume fraction of cylinders. Suppose that the end effects are negligible and the flow field inside the microchannel is fully developed. The cylindrical coordinates $(r, \theta, z)$ are adopted, with their origin located at the axis of the representative cylinder. Let $E_{z}=-(\partial \phi / \partial z)$ and $P_{z}=-(\partial P / \partial z)$ be respectively an applied electrical field and a pressure gradient; both are in the $z$-direction, $\phi$ and $P$ being respectively the electrical potential and the pressure. $U$ is the velocity of the electrolyte solution, which is a function of $r$ only.

\subsection{Electric field}

For the present problem, $\phi$ is described by

$$
\frac{1}{r} \frac{\partial}{\partial r}\left(r \frac{\partial \phi}{\partial r}\right)+\frac{\partial^{2} \phi}{\partial z^{2}}=-\frac{\rho_{\mathrm{el}}}{\varepsilon},
$$

where $\varepsilon$ is the dielectric constant of the electrolyte solution and $\rho_{\mathrm{el}}$ is the space charge density. Since $\phi=\phi(r, z)=\psi(r)-z E_{z}$, where $\psi(r)$ is the electrical potential in the absence of the applied electrical potential, or the equilibrium potential, Eq. (1) becomes

$\frac{1}{r} \frac{\mathrm{d}}{\mathrm{d} r}\left(r \frac{\mathrm{d} \psi}{\mathrm{d} r}\right)=-\frac{\rho_{\mathrm{el}}}{\varepsilon}$.

For the case of 1:1 electrolytes and low electrical potential, it can be shown that this expression reduces to

$\frac{1}{r} \frac{\mathrm{d}}{\mathrm{d} r}\left(r \frac{\mathrm{d} \psi}{\mathrm{d} r}\right)=\kappa^{2} \psi$,

where $\kappa=\left(2 I F^{2} / \varepsilon R T\right)^{1 / 2}$ is the Debye-Hückel parameter, $I$ is the ionic strength, $F$ and $R$ are respectively the Faraday constant and the gas constant, and $T$ is the absolute temperature. We assume the following boundary conditions:

$$
\begin{aligned}
& \psi(\mathrm{a})=\psi_{\mathrm{s}}, \\
& \frac{\mathrm{d} \psi}{\mathrm{d} r}(r=a \alpha)=0 .
\end{aligned}
$$

The first condition implies that the surface of a cylinder is remained at the constant potential $\psi_{\mathrm{s}}$, and the second condition arises from the nature of a cell model; that is, all physical properties reach their bulk values on the virtue surface $r=\alpha a$. The solution to Eq. (3) subject to Eqs. (4) and (5) is

$\psi(r)=\psi_{\mathrm{s}}\left[A I_{0}(\kappa r)+B K_{0}(\kappa r)\right]$,

where

$$
\begin{aligned}
& A=\frac{K_{1}(\kappa a \alpha)}{I_{0}(\kappa a) K_{1}(\kappa a \alpha)+I_{1}(\kappa a \alpha) K_{0}(\kappa a)}, \\
& B=\frac{I_{1}(\kappa a \alpha)}{I_{0}(\kappa a) K_{1}(\kappa a \alpha)+I_{1}(\kappa a \alpha) K_{0}(\kappa a)} .
\end{aligned}
$$

In these expressions, $I_{0}$ is the zero-order modified Bessel function of the first kind, $I_{1}$ is the first-order modified Bessel function of the first kind, $K_{0}$ is the zero-order modified Bessel function of the second kind, and $K_{1}$ is the first-order modified Bessel function of the second kind. 


\subsection{Flow field}

We assume that the electrolyte solution is an incompressible Newtonian fluid of constant physical properties, the Reynolds number is small, and the effect of gravity can be neglected. Therefore, the flow field can be described by

$\mu \frac{1}{r} \frac{\mathrm{d}}{\mathrm{d} r}\left[r \frac{\mathrm{d} U}{\mathrm{~d} r}\right]=\frac{\mathrm{d} p}{\mathrm{~d} z}-\rho_{\mathrm{el}} E_{z}$,

where $\mu$ is the viscosity of the electrolyte solution. Since

$$
\begin{aligned}
\rho_{\mathrm{el}} & =-2 n_{\infty} \exp \left(\frac{F \psi}{R T}\right) \\
& =-\varepsilon \kappa^{2} \psi_{\mathrm{s}}\left[A I_{0}(\kappa r)+B K_{0}(\kappa r)\right],
\end{aligned}
$$

Eq. (9) becomes

$$
\mu \frac{1}{r} \frac{\mathrm{d}}{\mathrm{d} r}\left[r \frac{\mathrm{d} U}{\mathrm{~d} r}\right]=\frac{\mathrm{d} p}{\mathrm{~d} z}+\varepsilon \psi_{\mathrm{s}} \kappa^{2} E_{z}\left[A I_{0}(\kappa r)+B K_{0}(\kappa r)\right] .
$$

The following boundary conditions are assumed for the flow field:

$$
\begin{aligned}
& U(a)=0, \\
& \frac{\mathrm{d} U}{\mathrm{~d} r}(r=a \alpha)=0 .
\end{aligned}
$$

The first condition implies that the outer surface of a cylinder is no-slip, and the second condition arises from the nature of a cell model. The solution to Eq. (11) subject to Eqs. (12) and (13) is

$$
\begin{aligned}
U(r)= & P_{z}\left(\frac{a^{2}}{4 \mu}\right)\left[1-\left(\frac{r}{a}\right)^{2}+2 \alpha^{2} \ln \left(\frac{r}{a}\right)\right] \\
& +\frac{\varepsilon \psi_{\mathrm{s}} E_{z}}{\mu}\left[A I_{0}(\kappa r)+B K_{0}(\kappa r)-1\right] .
\end{aligned}
$$

\subsection{Residence time distribution}

If a pressure gradient is not applied, $P_{z}=0$, Eq. (14) leads to

$t=\frac{L}{U(r)}=\frac{L}{C\left[A I_{0}(\kappa r)+B K_{0}(\kappa r)-1\right]}$,

where $C=\varepsilon \psi_{\mathrm{s}} E_{z} / \mu$. Differentiating this expression yields

$\mathrm{d} t=-\frac{L C\left(\kappa A I_{1}(\kappa r)-\kappa B K_{1}(\kappa r)\right)}{[U(r)]^{2}} \mathrm{~d} r$.

The residence time distribution, $E(t)$, can be expressed as

$E(t)=\frac{\mathrm{d} V}{V_{\mathrm{E}}} \frac{1}{\mathrm{~d} t}=\frac{U(r) 2 \pi r \mathrm{~d} r}{V_{\mathrm{E}}} \frac{1}{\mathrm{~d} t}$,

where $V_{\mathrm{E}}$ is the volumetric flow rate when only an electric field is applied. Substituting Eq. (16) into this expression, we obtain

$E(t)=-\frac{[U(r)]^{3} 2 \pi r}{C L V_{\mathrm{E}}\left[\kappa A I_{1}(\kappa r)-\kappa B K_{1}(\kappa r)\right]}$,

where the total volumetric flow rate $V_{\mathrm{E}}$ can be expressed as

$$
V_{\mathrm{E}}=C \frac{2 \pi}{\kappa}\left\{A\left[a \alpha I_{1}(\kappa a \alpha)-a I_{1}(\kappa a)\right]\right.
$$

$$
\left.-B\left[a \alpha K_{1}(\kappa a \alpha)-a K_{1}(\kappa a)\right]-\frac{\kappa}{2}\left((a \alpha)^{2}-a^{2}\right)\right\} .
$$

If a pressure gradient $P_{z}$ is applied, Eq. (14) yields

$t=\frac{L}{U(r)}=\frac{L}{P_{z}\left(\frac{a^{2}}{4 \mu}\right)\left[1-\left(\frac{r}{a}\right)^{2}+2 \alpha^{2} \ln \left(\frac{r}{a}\right)\right]+C\left[A I_{0}(\kappa r)+B K_{0}(\kappa r)-1\right]}$.

Differentiating this expression with respect to $r$ gives

$\mathrm{d} t=-L \frac{C\left[A \kappa I_{1}(\kappa r)-B \kappa K_{1}(\kappa r)\right]+P_{z} \frac{(a \alpha)^{2}}{2 \mu}\left[\frac{1}{r}-\frac{r}{(a \alpha)^{2}}\right]}{(U(r))^{2}} \mathrm{~d} r$.

Substituting this expression into Eq. (17) yields

$E(t)=-\frac{2 \pi r[U(r)]^{3}}{L V_{t}\left\{C\left[A \kappa I_{1}(\kappa r)-B \kappa K_{1}(\kappa r)\right]+P_{z} \frac{(a \alpha)^{2}}{2 \mu}\left[\frac{1}{r}-\frac{r}{(a \alpha)^{2}}\right]\right\}}$.

In this case, the total volumetric flow rate $V_{\mathrm{t}}$ can be expressed as

$V_{\mathrm{t}}=V_{\mathrm{E}}+\frac{\pi a^{4}}{8 \mu} P_{z}\left[4 \alpha^{4} \ln \alpha-3 \alpha^{4}+4 \alpha^{2}-1\right]$

\section{Results and discussion}

The influences of the key parameters of the system under consideration, including $\kappa a, E_{z}$, and $P_{z}$, on the flow field and the corresponding residence time distribution are investigated through numerical simulation. For illustration, the ranges of these parameters are $[0.001,400],\left[2.56 \times 10^{5}, 2.56 \times\right.$ $\left.10^{6} \mathrm{~V} / \mathrm{m}\right]$, and $\left[0,10^{3} \mathrm{~N} / \mathrm{m}^{3}\right]$ respectively. Also, we assume that $a=10^{-7} \mathrm{~m}, L=10^{-3} \mathrm{~m}, \psi_{\mathrm{s}}=25.6 \mathrm{mV}, T=298 \mathrm{~K}$, $N_{0}=6.022169 \times 10^{23} \mathrm{~mol}^{-1}, F=96,487 \mathrm{C}, \sum \varepsilon=7.08 \times$ $10^{-10} \mathrm{C} /(\mathrm{V} \mathrm{m})$, and $\mu=1 \times 10^{-3} \mathrm{~kg} /(\mathrm{m} \mathrm{s})$.

Fig. 2 illustrates the variations of velocity distribution $U(R)$ at various values of $\kappa a$. This figure reveals that $U$ increases with the increase in $\kappa a$; that is, the thinner the double layer (higher electrolyte concentration), the faster the flow of electrolyte solution. This is expected since the higher the concentration of electrolytes the greater the driving force for electroosmotic

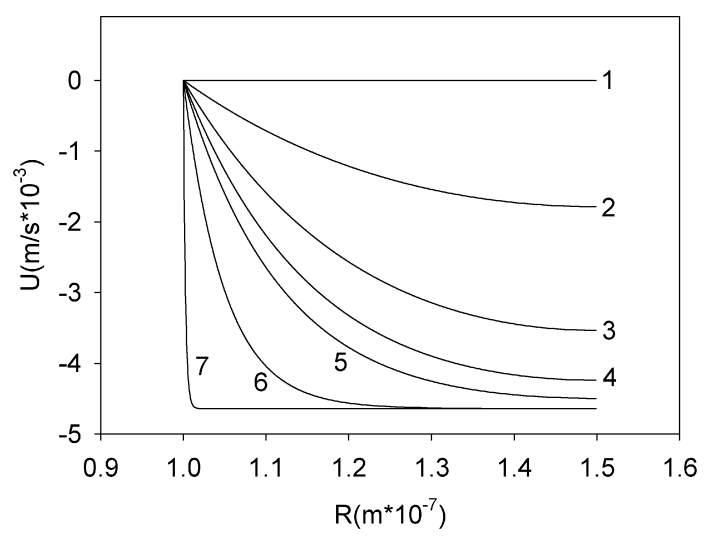

Fig. 2. Variation of fluid velocity $U(R)$ at various values of $\kappa a$ for the case where $E_{z}=2.56 \times 10^{5} \mathrm{~V} / \mathrm{m}, P_{z}=0 \mathrm{~N} / \mathrm{m}^{3}, \psi_{\mathrm{s}}=25.6 \mathrm{mV}$, and $\alpha=1.5$. Curve 1: $\kappa a=0.001 ; 2: \kappa a=2 ; 3: \kappa a=4 ; 4: \kappa a=6 ; 5: \kappa a=8 ; 6: \kappa a=20$; 7: $\kappa a=400$. 


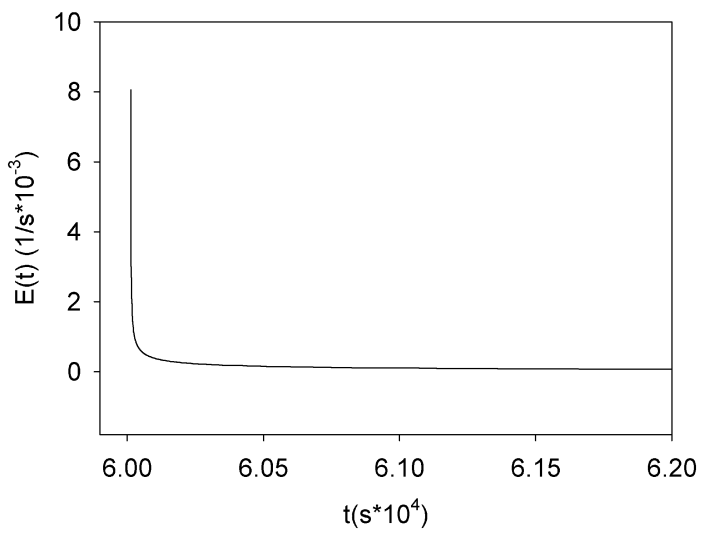

(a)

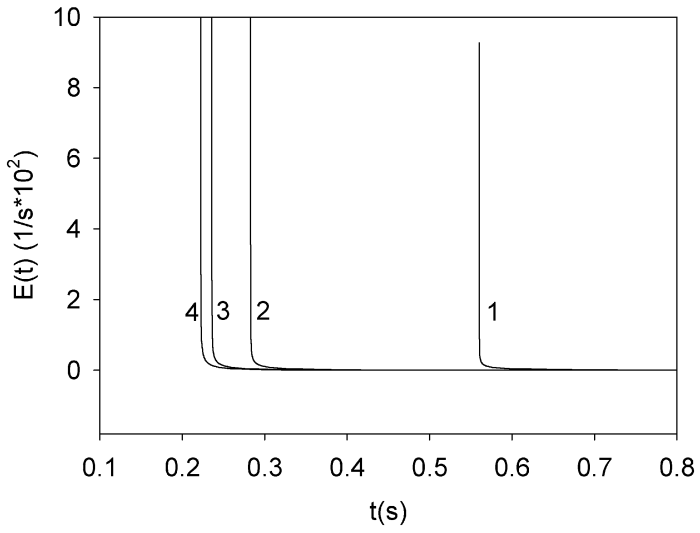

(b)

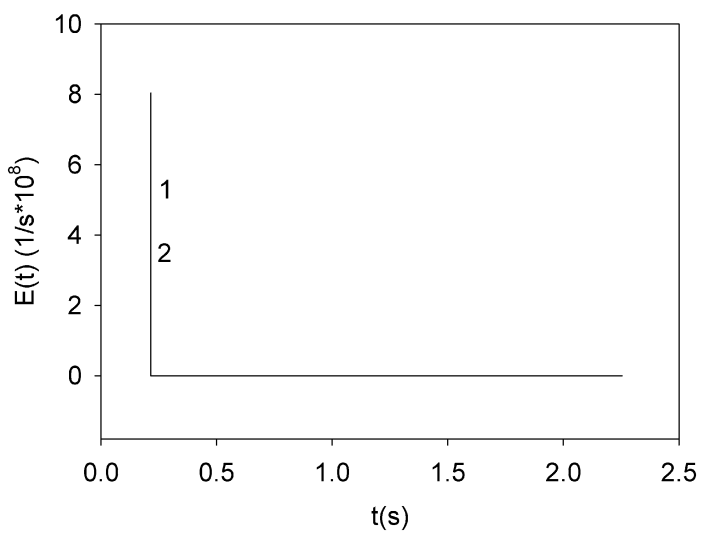

(c)

Fig. 3. Variation of residence time distribution $E(t)$ for the case when $E_{z}=2.56 \times 10^{5} \mathrm{~V} / \mathrm{m}, P_{z}=0 \mathrm{~N} / \mathrm{m}^{3}, \psi_{\mathrm{s}}=25.6 \mathrm{mV}$, and $\alpha=1.5$. (a) $\kappa a=0.005$; (b) curve 1: $\kappa a=2$; curve 2: $\kappa a=4$; curve 3: $\kappa a=8$; and curve 4: $\kappa a=10$; (c) curve 1: $\kappa a=100$; and curve 2: $\kappa a=400$.

flow. Also, the hydrodynamic retardation due to the presence of the double layer declines with its thickness. For the case when $\kappa a \ll 1$, the space between cylinders is filled with the double layer and the distribution of ionic species is uniform; so is $U(R)$. If $1 \leqslant \kappa a \leqslant 10, U(R)$ is close to a parabolic curve, and if $\kappa a$ is sufficiently large, it becomes flat again. If $\kappa a \rightarrow \infty$, it can be shown that Eq. (14) reduces to

$U=-\frac{\varepsilon \psi_{\mathrm{s}} E_{z}}{\mu}$.

Fig. 3 shows the variations of residence time distribution $E(t)$ at various values of $\kappa a$. As can be seen in Figs. $3 \mathrm{a}$ and $3 \mathrm{~b}$, if $\kappa a \ll 1$, the velocity distribution is parabolic, the flow field is close to a laminar flow, and it can be shown that

$E(t)=-\frac{2 \pi r L^{2}}{C V_{\mathrm{E}}\left[\kappa A I_{1}(\kappa r)-\kappa B K_{1}(\kappa r)\right]} \frac{1}{t^{3}}$.

Fig. $3 \mathrm{c}$ shows that for $1<\kappa a<10$, the larger the value of $\kappa a$, the faster the electroosmotic velocity, and the shorter the residence time. Note that $E(t)$ is concentrated near the shortest residence time. This is because if $\kappa a$ is sufficiently large, since the double layer surrounding a cylindrical reactor is thin, the distribution of fluid velocity becomes uniform at a distance not far from the surface of the cylindrical reactor. Note that $E(t)$ is able to extend to infinity because the fluid velocity vanishes on the surface of a cylindrical reactor. In Fig. 3d, $\alpha a \gg 1$, the fluid velocity approaches a uniform distribution, and $E(t)$ approaches a Dirac delta function.

The velocity distribution $U(R)$ and variations of residence time distribution $E(t)$ for various combinations of the scaled applied electric field $E^{*}=E_{z} /(R T / F L)$ and $\kappa a$ are illustrated in Figs. 4 and 5. In general, for a fixed double-layer thickness, the stronger the applied electric field, the greater the driving force for the electroosmotic flow, the faster the velocity distribution, and the shorter the residence time. Fig. 4a shows that for stronger $E^{*}, U(R)$ is faster, which causes a narrower $E(t)$ in Fig. 5a, with a larger maximum and a wider range. The double layer is thin in Figs. $4 \mathrm{~b}$ and $5 \mathrm{~b}$, and the corresponding $E(t)$ is close to a Dirac delta function. The influence of $E^{*}$ on $E(t)$ is similar to that in Fig. 5a. A comparison between Figs. 3 and 5 reveals that as $\kappa a$ (or the thickness of the double layer) varies, because both the shape and the magnitude of velocity distribution vary, the minimum of $E(t)$ and its shape vary accordingly. On the other hand, while the magnitude of velocity is influenced by $E^{*}$, the shape of the velocity distribution remains the same, and therefore, the residence time and the value of $E(t)$ are influenced by $E^{*}$, but the shape of $E(t)$ remains similar.

The influence of parameter $\alpha$ on the velocity distribution is presented in Fig. 6. Recall that $\alpha$ is a measure for the volume fraction of cylinders. Through adjustment of this parameter, both the space available for fluid flow and the number of cylinders can be designed. In general, the smaller the value of $\alpha$, the 


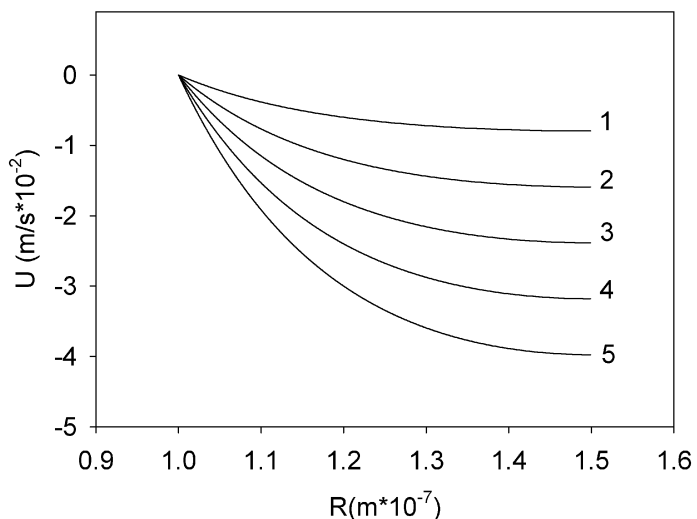

(a)

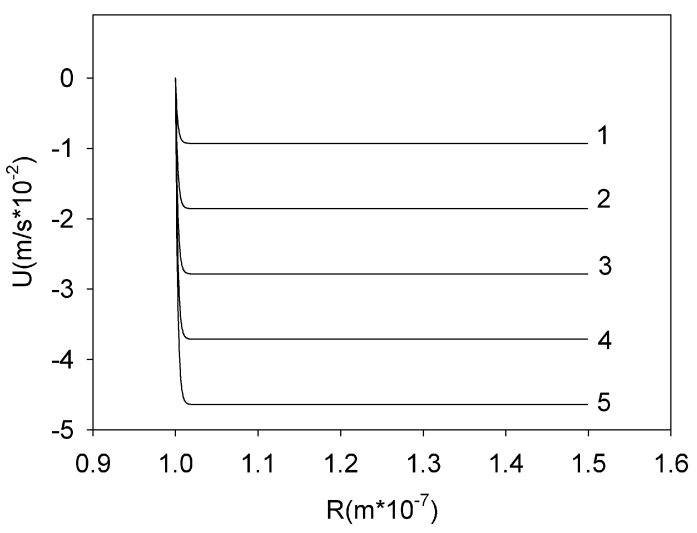

(b)

Fig. 4. Variation of fluid velocity $U(R)$ at various scaled applied electric fields $E^{*}$ for different $\kappa a$ for the case when $\psi_{\mathrm{s}}=25.6 \mathrm{mV}, P_{z}=0 \mathrm{~N} / \mathrm{m}^{3}$, and $\alpha=1.5$. (a) $\kappa a=5$, (b) $\kappa a=400$. Curve 1: $E^{*}=2$; curve 2: $E^{*}=4$; curve 3: $E^{*}=6$; curve 4: $E^{*}=8$; and curve $5: E^{*}=10$.

lower the mean fluid velocity is. This is because the smaller the value of $\alpha$, the smaller is the space available for fluid flow. As can be seen in Fig. 6 a, if $\alpha a \ll 1$, both the velocity distribution and the maximum velocity are influenced by the value of $\alpha$. If $\alpha$ is small, the velocity distribution is uniform, and it becomes parabolic when $\alpha$ is sufficiently large. The maximum velocity increases with the increase in $\alpha$. This is because if $\alpha a \ll 1$, the double layer surrounding a cylinder is thick, and if it is comparable to the gap between two cylinders, the velocity distribution become uniform. Also, the larger the value of, $\alpha$ the more space is available for fluid flow, and therefore, it is more possible for fluid velocity to reach a higher value. The general trends of velocity distribution shown in Fig. 6b, where $1<\alpha a<10$, are similar to those seen in Fig. 6a. However, a comparison between these two figures indicates that, because the double layer in Fig. 6b is thinner than that in Fig. 6a, the velocity distribution of the former reaches the boundary value faster than that of the latter does. As can be seen in Fig. $6 \mathrm{c}$, if $\alpha a \gg 1$, the double layer is very thin, and the velocity distribution becomes insensitive to the variation of $\alpha$.

The influences of the applied pressure gradient $P_{z}$ on the velocity distribution and the corresponding residence time distribution are shown in Figs. 7 and 8. Intuitively, the greater the pressure gradient, the faster the flow of liquid and the shorter the residence time is. These are observed in Figs. 7a and 8a, where

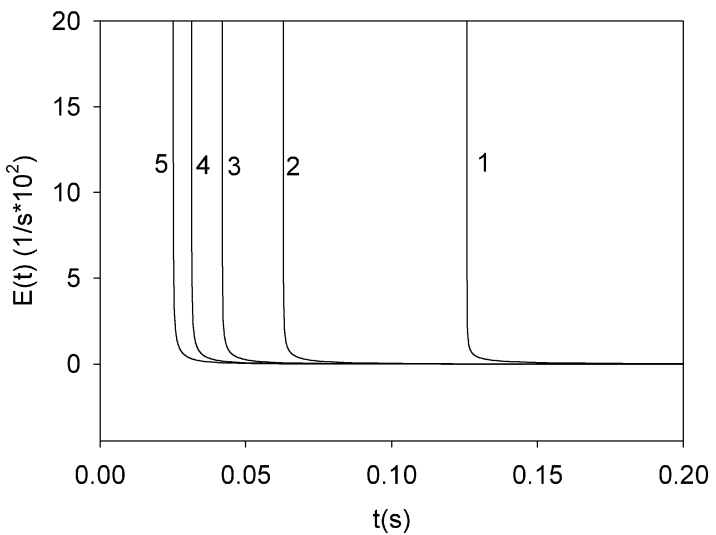

(a)

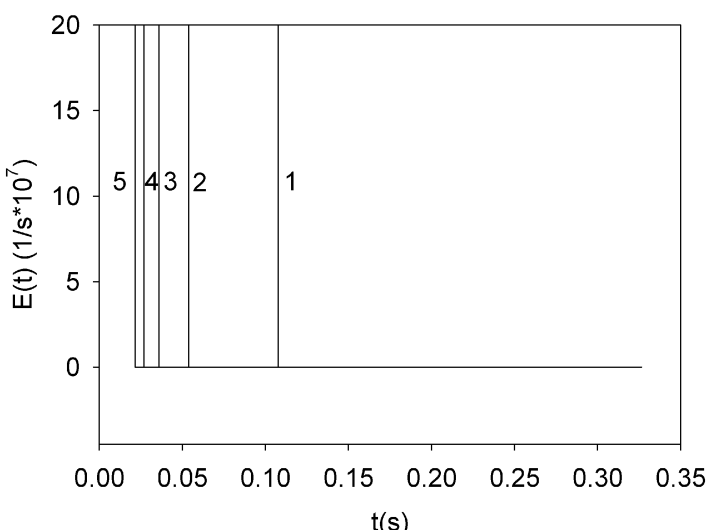

(b)

Fig. 5. Variation of residence time distribution $E(t)$ at various scaled applied electric fields $E^{*}$ for different $\kappa a$ for the case when $\psi_{\mathrm{s}}=25.6 \mathrm{mV}$, $P_{z}=0 \mathrm{~N} / \mathrm{m}^{3}$, and $\alpha=1.5$. (a) $\kappa a=5$, (b) $\kappa a=400$. Curve $1: E^{*}=2$; curve 2: $E^{*}=4$; curve $3: E^{*}=6$; curve $4: E^{*}=8$; and curve $5: E^{*}=10$.

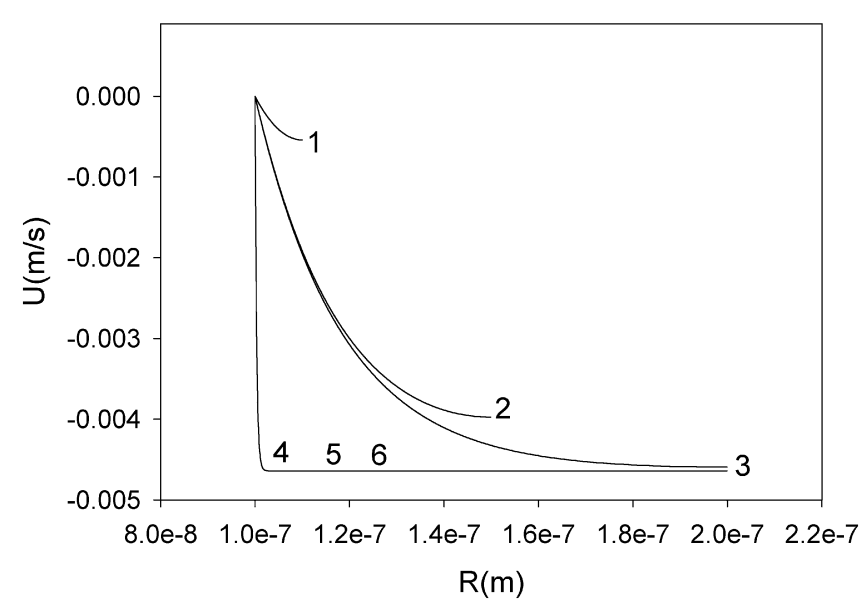

Fig. 6. Variation of fluid velocity $U(R)$ at different values of $\kappa a$ for the case when $E_{z}=2.56 \times 10^{5} \mathrm{~V} / \mathrm{m}, P_{z}=0 \mathrm{~N} / \mathrm{m}^{3}$, and $\psi_{\mathrm{s}}=25.6 \mathrm{mV}$. Curves 1-3: $\kappa a=4 ; 4-6: \kappa a=300$. Curves 1 and $4: \alpha=1.1 ; 2$ and $5: \alpha=1.5 ; 3$ and 6 : $\alpha=2.0$.

the double layer is thick. However, as illustrated in Figs. 7b and $8 \mathrm{~b}$, if the double layer is thin, both $U(R)$ and $E(t)$ become insensitive to the variation of $P_{z}$. This is because for the present microsystem, the driving force for fluid flow provided by the pressure gradient is limited, and the correspond- 


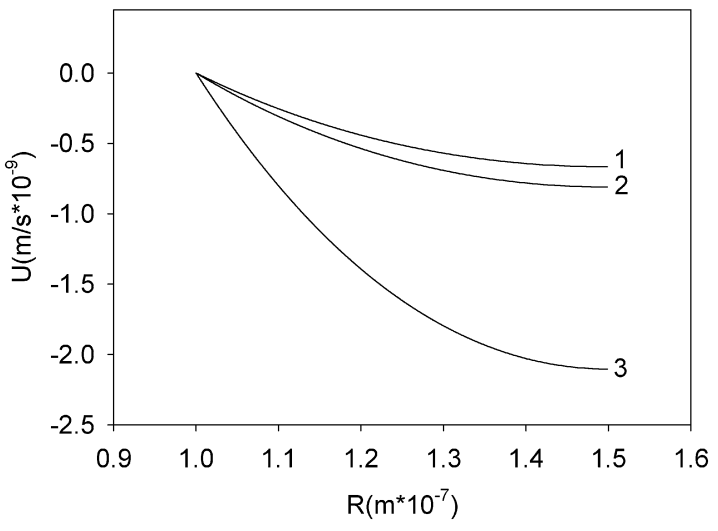

(a)

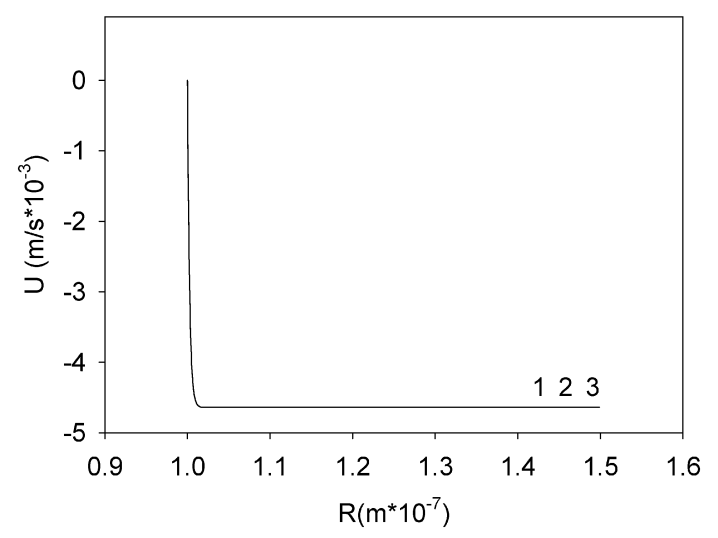

(b)

Fig. 7. Variation of fluid velocity $U(R)$ at various applied pressure gradients $P_{z}$ for the case when $E_{z}=2.56 \times 10^{5} \mathrm{~V} / \mathrm{m}, \psi_{\mathrm{s}}=25.6 \mathrm{mV}$, and $\alpha=1.5$. (a) $\kappa a=0.001$, (b) $\kappa a=400$. Curve $1: P_{z}=0 \mathrm{~N} / \mathrm{m}^{3}$; curve $2: P_{z}=$ $-10^{2} \mathrm{~N} / \mathrm{m}^{3}$; and curve $3: P_{z}=-10^{3} \mathrm{~N} / \mathrm{m}^{3}$.

ing fluid velocity is relatively low. However, since the driving force arising from the applied electric field is also small when the double layer is thick, the order of magnitude of fluid velocity is comparable to that when the flow is driven by the pressure gradient, and therefore, the influence of the pressure gradient is appreciable. If the double layer is thin, since the magnitude of the fluid velocity arising from the applied electric field is much greater than that arising from the pressure gradient, the influence of the pressure gradient becomes unimportant.

If the surface of a cylinder is remained at constant charge density, the boundary condition expressed in Eq. (4) needs to be replaced by

$\frac{\mathrm{d} \psi}{\mathrm{d} r}=-\frac{\rho_{\mathrm{s}}}{\varepsilon}, \quad r=a$,

where $\rho_{\mathrm{S}}$ denotes the surface charge density. It can be shown that

$$
\begin{aligned}
\psi(r)= & -\frac{\rho_{\mathrm{s}}}{\varepsilon}\left[D I_{0}(\kappa r)+E K_{0}(\kappa r)\right], \\
U(r)= & P_{z}\left[\frac{a^{2}}{4 \mu}\left(1-\left(\frac{r}{a}\right)^{2}\right)+\frac{(\alpha a)^{2}}{2 \mu} \ln \left(\frac{r}{a}\right)\right] \\
& -\frac{\rho_{\mathrm{s}} E_{z}}{\mu}\left[D I_{0}(\kappa r)+E K_{0}(\kappa r)-1\right],
\end{aligned}
$$

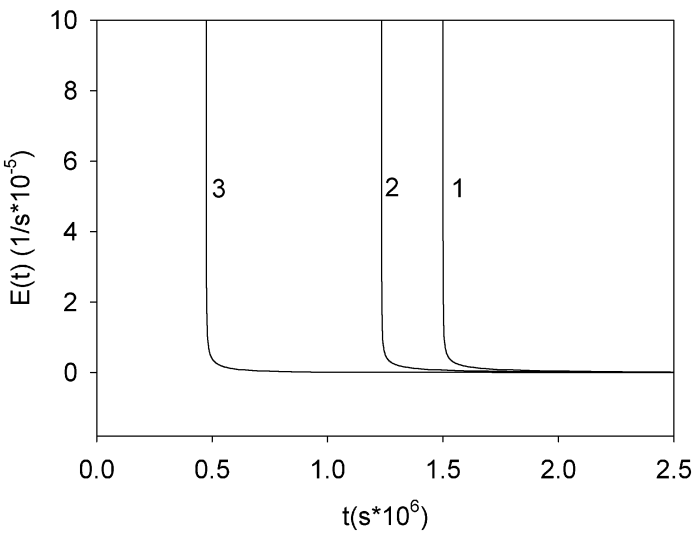

(a)

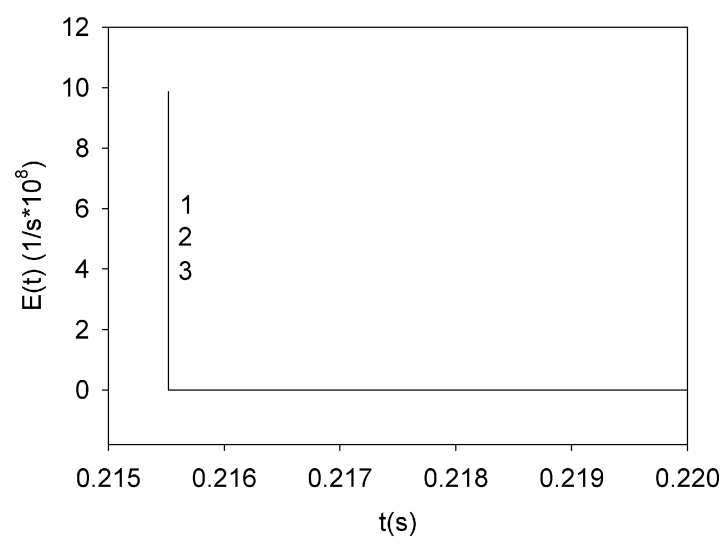

(b)

Fig. 8. Variation of residence time distribution $E(t)$ at various applied pressure gradients $P_{z}$ for the case when $E_{z}=2.56 \times 10^{5} \mathrm{~V} / \mathrm{m}, \psi_{\mathrm{s}}=25.6 \mathrm{mV}$, and $\alpha=1.5$. (a) $\kappa a=0.001$, (b) $\kappa a=400$. Curve $1: P_{z}=0 \mathrm{~N} / \mathrm{m}^{3}$; curve 2 : $P_{z}=-10^{2} \mathrm{~N} / \mathrm{m}^{3}$; and curve $3: P_{z}=-10^{3} \mathrm{~N} / \mathrm{m}^{3}$.

$$
E(t)=-\frac{2 \pi r[U(r)]^{3}}{L V_{t}\left\{C\left[D \kappa I_{1}(\kappa r)-E \kappa K_{1}(\kappa r)\right]+P_{z} \frac{(a \alpha)^{2}}{2 \mu}\left[\frac{1}{r}-\frac{r}{(a \alpha)^{2}}\right]\right\}},
$$

where $I_{1}$ is the first-order modified Bessel function of the first kind and $K_{1}$ is the first-order modified Bessel function of the second kind, and

$$
\begin{aligned}
& D=\frac{\left[K_{1}(\kappa \alpha a)\right]}{\kappa\left[I_{1}(\kappa a) K_{1}(\kappa \alpha a)-I_{1}(\kappa \alpha a) K_{1}(\kappa a)\right]}, \\
& E=\frac{\left[I_{1}(\kappa \alpha a)\right]}{\kappa\left[I_{1}(\kappa a) K_{1}(\kappa \alpha a)-I_{1}(\kappa \alpha a) K_{1}(\kappa a)\right]} .
\end{aligned}
$$

Also, Eq. (23) remains the same, except that in the $V_{\mathrm{E}}$ expressed in Eq. (19) the constants $A$ and $B$ need to be replaced by $C$ and $D$, respectively.

\section{Conclusions}

The flow and the electric fields for the flow of an electrolyte solution in a microchannel that comprises a bundle of cylinders are investigated theoretically under conditions of low surface potential. The system under consideration mimics, for example, a microreactor containing optical fibers. The governing electrokinetic equations are solved analytically and the results used to derive the residence time distribution, which is necessary 
for reactor design. The influence of the key parameters of the present system on the residence time distribution is examined through numerical simulation, and the results obtained reveal that the following could result in a shorter residence time: thin double layer, strong applied electric field, large applied pressure gradient, and small number of cylinders. The qualitative behavior of the residence time distribution is similar to that for the flow of an electrolyte solution in a cylindrical microchannel. The influence of the volume fraction of cylinders and the applied pressure gradient is important only if the double layer surrounding a cylinder is sufficiently thick.

\section{Acknowledgments}

This work is supported by the Department of Economics of the Republic of China under Grant 93-EC-17-A-09-S1-019 and the National Science Council of the Republic of China.

\section{References}

[1] G.F. Froment, K.B. Bischoff, Chemical Reactor Analysis and Design, Wiley, New York, 1990.

[2] H.S. Fogler, Elements of Chemical Reaction Engineering, Prentice-Hall, Upper Saddle River, NJ, 1999.

[3] O. Levenspiel, Chemical Reaction Engineering, Wiley, New York, 1999.

[4] K.D. Wise, Proc. IEEE 86 (1998) 1531.

[5] H. Löwe, W. Ehrfeld, Electrochim. Acta 21-22 (1999) 3679.

[6] S.C. Jacobson, T.E. Mcknight, J.M. Ramsey, Anal. Chem. 71 (1999) 4455.

[7] S.L.R. Barker, D. Ross, M.J. Tarlov, M. Gaitan, L.E. Locascio, Anal. Chem. 72 (2000) 5925.

[8] M.J. Mitchell, R. Qiao, N.R. Aluru, J. Microelectromech. Syst. 9 (2000) 435.

[9] A. Ajdari, Phys. Rev. E 65 (2001) 016301-1.

[10] L. Ren, D. Li, W. Qu, J. Colloid Interface Sci. 233 (2001) 12.

[11] P. Takhistov, K. Duginova, H.C. Chang, J. Colloid Interface Sci. 263 (2003) 133

[12] S. Ghosal, Annu. Rev. Fluid Mech. 38 (2006) 309.

[13] J.P. Hsu, T.H. Wei, J. Phys. Chem. B 109 (2005) 9160. 